\title{
4
}

\section{COMPETITIVIDAD EMPRESARIAL: CASO EMPRESAS PRIVADAS DE LA PROVINCIA DE SANTA ELENA.}

Jairo Cedeño, Verónica Benavides, Verónica Ponce. 


\title{
Competitividad Empresarial: Caso empresas privadas de la provincia de Santa Elena. \\ Business Competitiveness: Private companies case of the province of Santa Elena.
}

\author{
Jairo Cedeño Pinoargote, Verónica Benavides Benítez, Verónica Ponce Chalen \\ Universidad Estatal Península de Santa Elena. Carrera de Administración de Empresas \\ Campus La Libertad, vía principal Santa Elena - La Libertad - Ecuador \\ manuelcepi23@hotmail.com
}

\begin{abstract}
Resumen
El objetivo de esta investigación fue evaluar la competitividad empresarial de las empresas privadas de la provincia de Santa Elena, para identificar las ventajas competitivas que tienen a nivel nacional e internacional. La investigación plantea: el marco teórico relacionado con la competitividad empresarial, la técnica utilizada fue la encuesta, con el cuestionario como instrumento, los cuales fueron aplicados a los gerentes o administradores de las empresas privadas de la provincia de Santa Elena, permitiendo obtener la información requerida para el desarrollo de la investigación. Los resultados indicaron que las empresas privadas de la provincia de Santa Elena se encuentran preparadas para ser competitivas en el mercado local e internacional, para el efecto, se deberá fortalecer su competitividad, con la adquisición de programas de gestión, se debe puntualizar que en la parte administrativa existen debilidades, a su vez permitiendo una rápida y eficaz respuesta a las inquietudes sobre mejoramiento de las competencias.
\end{abstract}

Palabras Claves: Competitividad Empresarial; Competencia; Tecnóloga; Globalización; Calidad.

\begin{abstract}
The objective of the present investigation was to evaluate the business competitiveness of the private companies of the Province of Santa Elena in order to identify the competitive advantages they have at national and international level. The research proposes: the theoretical framework related to business competitiveness, the technique used was the survey, with the questionnaire as an instrument, which were applied to the managers or administrators of Private companies of the Province of Santa Elena allowing to obtain the required information For the development of research. The results indicated that the Private companies of the Province of Santa Elena are prepared to be competitive in the local and international market, therefore, it should strengthen that all of them are competitive, the acquisition of management programs, it should be pointed out that in The administrative side has weaknesses, in turn allowing a quick and effective response to the concerns about improving skills.
\end{abstract}

Keywords: Business competitiveness; Competition; technologist; globalization; Quality.

J. Cedeño/V. Benavides/V. Ponce. 


\section{Introducción}

La innovación tecnológica representa, en la actualidad, una de las fuentes para generar ventajas competitivas sostenibles en las empresas, porque es la fuerza motriz que impulsa la creación de riquezas, renueva las estructuras industriales y propicia el surgimiento de nuevos sectores productivos. Frente a la innovación de la empresa es difícil de administrar y evaluar.

La investigación permitió realizar una revisión de la literatura de los grandes pensadores como Adán Smith, David Ricardo, Karl Marx y Michael Porter, además de las bases teóricas como la ventaja absoluta, teoría del valor del trabajo y la ventaja competitiva de D. Ricardo.

La metodología de la investigación explica el tipo de estudio que se llevó a cabo, la caracterización de la muestra objeto de estudio, los instrumentos que se emplearon en la recolección de los datos, los recursos utilizados y finalmente se especifica el procedimiento. Además, se encuentran los resultados, discusión y las conclusiones de la investigación.

Arzola realizó un análisis comparativo entre las diversas metodologías hasta ahora utilizadas para medir el proceso de innovación tecnológica en las empresas, considerando las variables e indicadores más importantes en cada método. En esta investigación se definen los indicadores de la innovación tecnológica en general, los cuales incluyen la innovación organizativa. Presentando, además, valiosa información sobre los modos de evaluación de los resultados de la innovación.

Adam Smith, en su obra fundamental, la riqueza de las naciones, buscó explicar el crecimiento económico a partir del concepto de "división del trabajo", cuyo regulador es el mercado. La especialización de la fuerza de trabajo genera un incremento en la productividad que permite la acumulación de riquezas. Sin embargo, sólo aquellas economías orientadas hacia la producción para el intercambio son las beneficiarias, ya que las economías de subsistencias no son capaces de generar estas innovaciones que elevan el nivel de producción.

Esta división de la formación de valor de un producto en salario, renta y ganancia le permitió a Smith elaborar el concepto de "precio natural". El precio natural de los bienes (es decir, su valor de cambio) está dado por estos 3 factores, y cada uno de ellos tiene a su vez un nivel natural dentro del precio natural de los bienes. El precio natural es aquel que alcance para pagar, ni más ni menos, la renta de la tierra, el salario del trabajo y la ganancia del capital. Sin embargo, Smith observó que en los precios de los bienes en el mercado, no suele cumplirse con esa definición. El precio de mercado suele ser distinto al precio natural, y es a través de la competencia que se logra que estos niveles se acerquen. De esta forma, la acción del mercado hace que los precios alcancen un equilibrio.

David Ricardo muestra que la ganancia industrial se ve determinada por la ganancia agrícola, ya que los insumos de la industria provienen del excedente de la producción agrícola. Cuanta más producción agrícola haya, mayores serán las posibilidades de obtener ganancia en la actividad industrial porque los precios de los productos agrícolas son bajos.

Karl Marx argumenta: El valor de cambio es un requisito necesario para que pueda existir el cambio privado y la sociedad capitalista. Si bien existen diferencias en los valores de uso de los productos, el sistema ignora esas "diferencias materiales individuales" y crea una equivalencia general entre ellos; sólo a través de este proceso puede existir una sociedad capitalista con cambio privado. Con esta teoría del valor-trabajo, Marx se aleja de los economistas clásicos, porque el valor de cambio de un bien lo determina por la cantidad de trabajo necesario para producirlo, entonces el trabajo no tiene valor, ya que él mismo es el patrón que determina el valor de un producto, y no puede determinarse a sí mismo.

Cuando un capitalista compra fuerza de trabajo en el mercado, la paga por su valor de cambio, determinado por el tiempo de trabajo socialmente necesario que incorpora el trabajador en sus medios de subsistencia. Sin embargo, al utilizarla se apropia de su valor de uso, que genera un nuevo valor de cambio, y que además puede utilizarse por más tiempo que el necesario para producir los medios de subsistencia del trabajador. De esta diferencia entre el valor de cambio de la fuerza de trabajo y el valor de uso, el capitalista obtiene la "plusvalía" que le permite realizar acumulación de ganancias.

Sin este mecanismo, la apropiación de ganancias no sería posible, ya que el cambio de mercancías no podría generar excedentes por sí mismo. Por ejemplo, si una persona con una determinada cantidad de dinero cambia ese dinero por una mercancía, lo está cambiando por el valor de cambio de esa mercancía.

a). De acuerdo con el modelo de la ventaja competitiva de Porter, la estrategia competitiva toma acciones ofensivas o defensivas para crear una posición

\section{J. Cedeño/V. Benavides/V. Ponce.}


defendible en una industria, con la finalidad de hacer frente, con éxito, a las fuerzas competitivas y generar un retorno sobre la inversión. "la base del desempeño sobre el promedio dentro de una industria es la ventaja competitiva sostenible".

Una empresa posee una ventaja competitiva cuando tiene alguna característica diferencial respecto de sus competidores, que le confiere la capacidad para alcanzar unos rendimientos superiores a ellos, de manera sostenible en el tiempo.

La ventaja competitiva consiste en una o más características de la empresa, que puede manifestarse de muy diversas formas. Una ventaja competitiva puede derivarse tanto de una buena imagen, de una prestación adicional de un producto, de una ubicación privilegiada o simplemente de un precio más reducido que el de los rivales.

Este estudio realizado por Deloitte comprende un análisis de opinión realizado a 90 empresarios. Se utilizó como referencia el Reporte Global del World Economic Forum (WEF), el cual analiza el conjunto de las instituciones y factores que determinan el nivel de competitividad y la capacidad de producción de los países. El estudio del WEF se enfoca en 12 pilares y más de 100 indicadores concentrados en 3 segmentos: requerimientos básicos, factores impulsados por la eficiencia e innovación, obteniendo indicadores para más de 140 países a través de un ranking de competitividad. "b).- Para el año 2012, el Ecuador se ubicó en el ranking 86 de 144, lo cual significó una mejoría; 15 puestos sobre el año anterior. En el indicador de los requerimientos básicos el cual incluye una evaluación del desempeño de las instituciones, la infraestructura, ambiente macroeconómico, salud y educación primaria, el país se ubica en el puesto 75 (ranking 82 en el año 2011). El indicador de eficiencia, que abarca: educación superior, eficiencia de mercados de bienes, eficiencia de mercado laboral, desarrollo del mercado financiero, capacidad tecnológica y tamaño de mercado, el país alcanzó el puesto 100 (ranking 107 en el año anterior) y, por último, en el segmento de los factores de innovación el país obtuvo el puesto 93 (ranking 103 en el año 2011).

De acuerdo a los resultados obtenidos del estudio realizado por Deloitte, el $60 \%$ de los empresarios consideran que el nivel de competitividad del Ecuador no ha mejorado con respecto a los demás países de la región; mientras que el $40 \%$ consideran lo contrario. Es importante destacar que el año anterior, el 75\% de los encuestados consideraron un bajo nivel de competencia, lo cual refleja una perspectiva más optimista para el año 2012

J. Cedeño/V. Benavides/V. Ponce.
Según el World Economic Forum los mayores aumentos fueron para; México (subió 5 puestos), Perú (subió 6 puestos), Brasil (subió 5 puestos) y Panamá (subió 4 puestos), mientras que Ecuador se destaca (subió 15 puestos).

En cuanto a buenas prácticas de competitividad se refiere, a participantes como Colombia (69) y Perú (61), los cuales por su nivel de desarrollo son un referente en el talento humano, mercados financieros y de capitales, infraestructura y tecnología, entre otros.

\section{Materiales y métodos.}

Se llevó a cabo un estudio no experimental que en su primera fase fue evaluativa, este estudio puede clasificarse como una investigación evaluativa, pues su objeto es valorar y enjuiciar el diseño, ejecución, efectos, utilidades y grado de logro de los objetivos de programas, a fin de corregir las deficiencias e introducir los reajustes necesarios.

\section{Población}

La población de donde se obtuvo la información que permitió el desarrollo la investigación la constituían las 21 empresas privadas más grandes de la provincia de Santa Elena

Tabla 1: Empresas privadas Provincia de Santa Elena.

\begin{tabular}{|l|l|l|}
\hline No. & \multicolumn{1}{|c|}{ EMPRESAS } & \multicolumn{1}{c|}{ DIRECcIón } \\
\hline 1 & Servientrega & La Libertad \\
\hline 2 & Cooperativa FINCA & La Libertad \\
\hline 3 & Hotel Colón Miramar & Salinas \\
\hline 4 & Hotel las Conchas & Salinas \\
\hline 5 & Hotel Chipipe & Salinas \\
\hline 6 & Keramicos & La Libertad \\
\hline 7 & Lubricantes ALEX & La Libertad \\
\hline 8 & Damasco & La Libertad \\
\hline 9 & Mueblería Ideal & La Libertad \\
\hline 10 & Muebles \& Diseños & La Libertad \\
\hline 11 & Banco del Pichincha & La Libertad \\
\hline 12 & Banco Bolivariano & La Libertad \\
\hline 13 & Cooperativa Huancavilca & La Libertad \\
\hline 14 & Atrapen & La Libertad \\
\hline 15 & Disensa & La Libertad \\
\hline 16 & Yacht Club & Salinas \\
\hline 17 & Automotores Continental & Salinas \\
\hline 18 & Restaurante El Rincón & La Libertad \\
\hline
\end{tabular}




\begin{tabular}{|l|l|l|}
\hline 19 & $\begin{array}{l}\text { Noe "Shipping Agency } \\
\text { Cia" }\end{array}$ & La Libertad \\
\hline 20 & Edinca & La Libertad \\
\hline 21 & Industria Serviterra S.A. & Santa Elena \\
\hline
\end{tabular}

Fuente: Cámara de comercio de la Provincia de Santa Elena.

\section{Instrumentos}

La técnica aplicada fue la Encuesta, con el Cuestionario como instrumento, el cual fue aplicado a los gerentes o administradores de las empresas que se mencionaron anteriormente.

\section{Resultados}

El estudio se realizó a las empresas privadas de la provincia de Santa Elena. Se presentan de forma separada los resultados que corresponden a los aspectos que abarcan la competitividad empresarial, administrativa, comercial y logística; financiera, talento humano, tecnología; y, ambiente, se evaluaron diferentes temáticas y su aplicación en alta, buena o poca medida, de las cuales se analizaron aquellas que se consideraron como factores determinantes que detienen la competitividad en las empresas privadas de la provincia.

A continuación se presentan resultados:

Tabla 2.- ¿Cuál de las siguientes variables han sido definidas y controladas en la empresa?

\begin{tabular}{|c|c|c|c|c|c|}
\hline & Frecuencia & Porcentaje & $\begin{array}{c}\text { Porcentaje } \\
\text { válido }\end{array}$ & $\begin{array}{l}\text { Porcentaje } \\
\text { acumulado }\end{array}$ \\
\hline \multirow{7}{*}{ Válidos } & Misión & 1 & $4,8 \%$ & $6,7 \%$ & $6,7 \%$ \\
\hline & Objetivos & 2 & $9,5 \%$ & $13,3 \%$ & $20 \%$ \\
\hline & $\begin{array}{l}\text { Políticas } \\
\end{array}$ & 3 & $14,3 \%$ & $20 \%$ & $40 \%$ \\
\hline & $\begin{array}{c}\text { Resultado } \\
\mathrm{s}\end{array}$ & 2 & $9,5 \%$ & $13,3 \%$ & $53,3 \%$ \\
\hline & $\begin{array}{l}\text { Todos las } \\
\text { opciones }\end{array}$ & 3 & $14,3 \%$ & $20 \%$ & $73,3 \%$ \\
\hline & $\begin{array}{c}\text { Misión, } \\
\text { Visión, } \\
\text { Valores }\end{array}$ & 4 & $19 \%$ & $26,7 \%$ & $100 \%$ \\
\hline & Total & 15 & $71,4 \%$ & $100 \%$ & \\
\hline Perdidos & Sistema & 6 & $28,6 \%$ & & \\
\hline \multicolumn{2}{|c|}{ Total } & 21 & $100 \%$ & & \\
\hline
\end{tabular}

Al revisar la función administrativa se encontró con grandes deficiencias ya que solo el $20 \%$ conoce la misión, visión, valores, objetivos, políticas y resultados de la empresa, con lo cual se puede determinar que las empresas no son competitivas en la parte administrativa, lo cual limitaría el éxito de las empresas porque su administrador no tiene claro las metas de su organización

Tabla 3.- ¿La empresa cuenta con un área comercial claramente establecida?

J. Cedeño/V. Benavides/V. Ponce.

\begin{tabular}{|c|c|c|c|c|c|}
\hline \multicolumn{2}{|l|}{} & Frecuencia & Porcentaje & $\begin{array}{c}\text { Porcentaje } \\
\text { válido }\end{array}$ & Porcentaje acumulado \\
\hline Válidos & $\mathrm{Si}$ & 15 & 71,4 & $\mathbf{1 0 0 , 0}$ & $\mathbf{1 0 0 , 0}$ \\
\hline Perdidos & Sistema & 6 & 28,6 & & \\
\hline \multicolumn{2}{|c|}{ Total } & $\mathbf{2 1}$ & $\mathbf{1 0 0 , 0}$ & & \\
\hline
\end{tabular}

La mayoría de las empresas encuestadas de la provincia tienen un área comercial claramente establecida, lo cual es positivo

Tabla 4.- ¿Tiene herramientas implementadas para determinar cuál es la rentabilidad de su negocio?

\begin{tabular}{|c|c|c|c|c|c|}
\hline \multicolumn{2}{|l|}{} & Frecuencia & Porcentaje & $\begin{array}{c}\text { Porcentaje } \\
\text { válido }\end{array}$ & $\begin{array}{c}\text { Porcentaje } \\
\text { acumulado }\end{array}$ \\
\hline Válidos & $\mathrm{Si}$ & 15 & $71,4 \%$ & $\mathbf{1 0 0 \%}$ & $\mathbf{1 0 0 \%}$ \\
\hline Perdidos & Sistema & 6 & $28,6 \%$ & & \\
\hline \multicolumn{2}{|c|}{ Total } & $\mathbf{2 1}$ & $\mathbf{1 0 0 \%}$ & & \\
\hline
\end{tabular}

El total de los empresarios de la provincia de Santa Elena cuentan con una herramienta para medir la rentabilidad del negocio, lo cual es importante para conocer cuál ha sido el rendimiento de lo que se ha invertido en un tiempo determinado.

Tabla 5.- Considera usted que el nivel de satisfacción y motivación de su personal en el trabajo es:

\begin{tabular}{|c|c|c|c|c|c|}
\hline & & Frecuencia & Porcentaje & $\begin{array}{c}\text { Porcentaje } \\
\text { válido } \\
\end{array}$ & $\begin{array}{l}\text { Porcentaje } \\
\text { acumulado }\end{array}$ \\
\hline \multirow[t]{3}{*}{ Válidos } & Alto & 11 & $52,4 \%$ & $73,3 \%$ & $73,3 \%$ \\
\hline & Medio & 4 & $19,0 \%$ & $26,7 \%$ & $100 \%$ \\
\hline & Total & 15 & $71,4 \%$ & $100 \%$ & \\
\hline Perdidos & Sistema & 6 & $28,6 \%$ & & \\
\hline \multicolumn{2}{|c|}{ Total } & 21 & $100 \%$ & & \\
\hline
\end{tabular}

Un porcentaje alto de empresarios de la provincia de Santa Elena considera que el personal que labora en su compañía se encuentra altamente motivado y satisfecho con el trato laboral que ellos reciben, lo cual es positivo evidencia que lo necesita, por lo tanto, el compromiso que asume con la empresa será más productivo para la misma. 
Tabla 6.- La empresa mejora o adquiere cada 5 años sistemas de gestión (informática-hardware y software -, incorporación de personal cualificado, cursos de formación). ¿En qué nivel?

\begin{tabular}{|c|c|c|c|c|c|}
\hline \multicolumn{2}{|l|}{} & Frecuencia & Porcentaje & $\begin{array}{c}\text { Porcentaje } \\
\text { válido }\end{array}$ & $\begin{array}{c}\text { Porcentaje } \\
\text { acumulado }\end{array}$ \\
\hline Válidos & Alto & 7 & $33,3 \%$ & $46,7 \%$ & $46,7 \%$ \\
\hline & Moderado & 4 & $19 \%$ & $26,7 \%$ & $73,3 \%$ \\
\hline & Bajo & 4 & $19 \%$ & $26,7 \%$ & $\mathbf{1 0 0 \%}$ \\
\hline & Total & $\mathbf{1 5}$ & $\mathbf{7 1 , 4 \%}$ & $\mathbf{1 0 0 \%}$ & \\
\hline Perdidos & Sistema & 6 & $28,6 \%$ & & \\
\hline \multicolumn{2}{|c|}{ Total } & $\mathbf{2 1}$ & $\mathbf{1 0 0 \%}$ & & \\
\hline
\end{tabular}

Un porcentaje alto de los empresarios de la provincia Santa Elena han adquirido sistemas de gestión para ser competitivos en el mercado.

Tabla 7.- ¿La cultura y la estrategia de la compañía involucran aspectos, impactos y riesgos ambientales?

\begin{tabular}{|c|c|c|c|c|c|}
\hline & & Frecuencia & Porcentaje & $\begin{array}{c}\text { Porcentaje } \\
\text { válido }\end{array}$ & $\begin{array}{l}\text { Porcentaje } \\
\text { acumulado }\end{array}$ \\
\hline \multirow{3}{*}{ Válidos } & $\mathrm{Si}$ & 13 & $61,9 \%$ & $86,7 \%$ & $86,7 \%$ \\
\hline & No & 2 & $9,5 \%$ & $13,3 \%$ & $100 \%$ \\
\hline & Total & 15 & $71,4 \%$ & $100 \%$ & \\
\hline Perdidos & $\begin{array}{r}\text { Siste } \\
\\
\text { ma }\end{array}$ & 6 & $28,6 \%$ & & \\
\hline \multicolumn{2}{|c|}{ Total } & 21 & $100 \%$ & & \\
\hline
\end{tabular}

La cultura y la estrategia de la compañía referentes a los impactos y riesgos ambientales son considerados como parte de la estrategia de competitividad de las empresas.

\section{Discusión}

D"ARMAS (2000) bajo la misma modalidad anterior, realizó un estudio sectorial de competitividad de las empresas del área de Calderería en el estado de Bolívar, con el objeto de determinar las estrategias a seguir para lograr un incremento en la competitividad del área y en el desarrollo de las empresas bolivarense. Sobre la base de la información analizada, se definieron estrategias genéricas orientadas a mejorar la competitividad interna de las empresas del área de Calderería, y estrategias genéricas orientadas a mejorar los factores básicos del desarrollo.

El aporte de esta investigación está enmarcado en que las empresas privadas de la provincia de Santa Elena se encuentran preparadas para ser competitivas en el mercado local. Por lo tanto, hay que trabajar por parte de los empresarios en la adquisición de programas de gestión para estar a nivel de otras empresas locales. En este sentido, se debe puntualizar que en la parte administrativa existen debilidades muy fuertes, debido a que se desconocen los objetivos y metas de la organización. Como universidad se trabaja mucho en las estrategias de los impactos y riesgos ambientales con la responsabilidad social hacia la comunidad.

\section{Conclusiones}

Como resultado de la investigación se obtuvieron en síntesis las siguientes conclusiones:

- Los administradores de las empresas privadas de la provincia de Santa Elena deberían permanecer en sus áreas de trabajo un promedio de 5 años (según encuestas realizadas a los administradores) para poder cumplir la planificación y dejar a las empresas con una buena participación en el mercado.

- En lo comercial y logística, las mayorías de las empresas cuentan con un área comercial claramente establecidas.

- Las mayorías de las empresas tienen herramientas financieras implementadas para determinar cuál es la rentabilidad de du negocio.

- Se debe adquirir sistemas de gestión (informática-hardware y software incorporación de personal calificado, cursos de formación) porque en la actualidad el 38\% tienen un sistema bajo moderado y debería ser muy alto para ser competitivo.

- $\quad$ El 86,7\% de las empresas están involucrada en el impacto y riesgos ambientales.

- Con respecto a la organización, se da una radiografía de las empresas se encontró que el primer involucrado debe ser el personal, la tecnificación de sus equipos, la motivación, los incentivos, lo cual lleva a puntualizar que las 
empresas deben tener certificación calidad basado en ISO-9000.

- Por otro lado, las empresas deben trabajar hacia el mejoramiento continuo basado en sistema de calidad orientados a ser competitivas en el mercado. Para ello, los administradores deben tener claro que las empresas competitivas en el mercado son las que van a superar las crisis.

\section{Referencias}

(1) ARZOLA, MINERVA. (1999). Trabajo de Ascenso; "Variables e indicadores para la gestión estratégica del proceso de innovación tecnológica en las empresas". Puerto Ordaz (Venezuela): U.N.E.X.P.O.

(2) D"ARMAS REGNAULT, MAYRA JOSE (2000). "Diseño de un plan de estrategias para mejorar la competitividad del sector bienes de capital en el área de Calderia del estado Bolivar". Puerto Ordaz (Venezuela) U.N.E.

(3) DELOITTE. (2013) Ecuador competitivo

(4) MARX, K El capital, Tomo I

(5) PORTER, M. (1990): The Competitive Advantage of Nations. Nueva York. Free Press.
(6) PORTER, M. (2007) Ventaja Competitiva.

(7) RICARDO, D. 1817. Principios de economía política y tributación. Ed. F.C.E, México 1973.

(8) SMITH, A. Los clásicos: Adam Smith y la ventaja absoluta.

(9) SMITH A. (1987) Los clásicos: Adam Smith y la ventaja absoluta. 